\title{
RSD: A Metric for Achieving Range-Free Localization beyond Connectivity
}

\author{
Ziguo Zhong and Tian He, Member, IEEE
}

\begin{abstract}
Wireless sensor networks have been considered as a promising tool for many location-dependent applications. In such deployments, the requirement of low system cost prohibits many range-based methods for sensor node localization; on the other hand, range-free approaches depending only on radio connectivity may underutilize the proximity information embedded in neighborhood sensing. In response to these limitations, this paper introduces a proximity metric called RSD to capture the distance relationships among 1-hop neighboring nodes in a range-free manner. With little overhead, RSD can be conveniently applied as a transparent supporting layer for state-of-the-art connectivity-based localization solutions to achieve better accuracy. We implemented RSD with three well-known algorithms and evaluated using two outdoor test beds: an 850-foot-long linear network with 54 MICAz motes, and a regular 2D network covering an area of 10,000 square feet with 49 motes. Results show that our design helps eliminate estimation ambiguity with a subhop resolution, and reduces localization errors by as much as 35 percent. In addition, simulations confirm its effectiveness for large-scale networks and reveal an interesting feature of robustness under unevenly distributed radio path loss.
\end{abstract}

Index Terms-Wireless sensor networks, localization, range free, neighborhood sensing, signature distance, RSD.

\section{INTRODUCTION}

W IRELESS sensor networks (WSN) have been proposed for many location-sensitive applications [1], such as battlefield monitoring [2], environment surveillance [3], object tracking [4], etc. In addition, many routing and management protocols [5], [6] proposed for such networks are built on the assumption that geographic parameters of sensor nodes are available. Although node localization plays an important role in all those systems, itself is a challenging problem due to the severe resource constraints at each low-cost and tiny sensor node.

Many ideas have been proposed for node localization in WSN. Based on whether accurate ranging is required, there are basically two types of methods: 1) range-based, and 2) range-free. Range-based methods can be accurate, but costly for requiring per-node ranging hardware [8], [9], [12], [14], [20], extensive system calibration or environment profiling [7], [10], [38], which may not be possible for largescale outdoor sensor networks. Range-free approaches localize nodes based on simple sensing, such as wireless connectivity [24], [25], [27], [30], [31], anchor proximity [23], [26], [28], and in-field event detections [34], [35], [36], [46]. Among them, solutions applying radio connectivity feature a low system cost, however, by sacrificing localization accuracy. Li et al. [39], [40] made early contributions to improve connectivity-based methods by proposing the idea of sorted radio signal strength quantization that features a simple system design, but under the assumption of a preknown and uniform 1-hop radio range throughout the network.

- The authors are with the Department of Computer Science and Engineering, University of Minnesota, 200 Union Street SE, Minneapolis, MN 55455. E-mail: \{zhong, tianhe\}@cs.umn.edu.

Manuscript received 23 Nov. 2009; revised 13 Dec. 2010; accepted 1 Feb. 2011; published online 17 Mar. 2011.

Recommended for acceptance by J. Cao.

For information on obtaining reprints of this article, please send e-mail to: tpds@computer.org, and reference IEEECS Log Number TPDS-2009-11-0578. Digital Object Identifier no. 10.1109/TPDS.2011.105.
The work in this paper is motivated from the finding that localization by means of mere connectivity may underutilize the proximity information available from neighborhood sensing. Although radio signal strength (RSS) is considered irregular in many cases [37], [38], [41], [42], for unknown radio propagation loss, multipath effects, hardware discrepancy, and so forth, our empirical study shows that in outdoor open-air scenarios, RSS weakens approximately monotonically with increasing physical distance, especially from the viewpoint of a single node, which might provide the useful information about which neighboring node is closer and which is further.

Following this finding, we propose the regulated signature distance, or RSD for short, as a metric of proximity among 1hop neighboring nodes. RSD brings in a novel range-free approach to extracting the relative distance information from neighborhood orderings easily obtained from RSS sensing. With little overhead, RSD can be conveniently embedded in connectivity-based localization algorithms to improve accuracy. We have augmented three such algorithms (MDS-MAP [24], DV-Hop [25], and RPA [31]) with RSD, and evaluated in two types of outdoor test beds: an 850-foot-long linear network with $54 \mathrm{MICAz}$ motes, and a 2D network covering an area of 10,000 square feet with 49 motes. Results show noticeable performance gains including eliminating ambiguity and reducing localization errors by as much as 35 percent. Besides, simulations confirm the effectiveness of RSD for large-scale networks, and reveal an interesting feature of robustness to unevenly distributed radio path loss.

In the following, Section 2 starts with empirical data analysis. Section 3 gives the design and Section 4 reports testbed experiments. Due to space constraints, we provide related work and simulation results in Appendices 1 and 9, which can be found on the Computer Society Digital Library at http://doi.ieeecomputersociety.org/10.1109/TPDS. 2011.105, respectively. And Section 5 concludes the paper. 


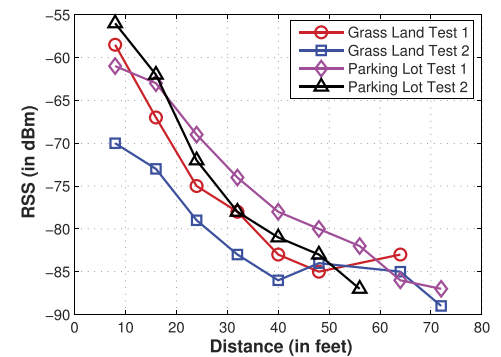

Fig. 1. Experimental results: RSS versus distance.

\section{Empirical Data as Motivation}

This paper is motivated by the experimental data showing the following in the outdoor open-air environments:

- $\quad$ network-wide monotonic relationship between RSS and physical distance does not hold, but

- per-node monotonic RSS-distance relationship holds well, i.e., any single node's RSS sensing results for neighboring nodes can be used as an indicator for the relative "near-far" relationship among them.

In the following, we explain results from a preliminary test as well as a large-scale experiment for verification.

\subsection{Preliminary Experiments}

Fig. 1 shows RSS results from MICAz nodes tested in two outdoor environments: grass land and parking lot. We placed nine sender nodes at various distances from a receiver node. Each sender broadcast 100 packets with $0 \mathrm{dBm}$ TX power, and the receiver node recorded RSS upon receiving a packet. In the grass-land scenario, we conducted the test twice with different receiver nodes placed at the same location without moving or switching sender nodes (Grass Land Tests 1 and 2). In the parking lot scenario, identical sets of nodes were tested during daytime (Parking Lot Test 1) and at night (Parking Lot Test 2). Tests were conducted multiple times and the curve patterns did not show significant change.

Fig. 1 states that at the system level, using RSS for distance estimation is not reasonable, since identical RSS values may correspond to different distances. However, for individual curve (i.e., from the viewpoint of a single node), RSS values mostly decreased monotonically with increasing distance, conveying information about relative "near-far" relationships among 1-hop neighbors.

\subsection{Large-Scale Experiments}

We then conducted two large-scale outdoor experiments to verify the above observation. The first was a linear network with $54 \mathrm{MICAz}$ nodes covering an 850-foot-long road segment. In the second experiment, we constructed a gridshaped 2D network occupying an area of 10,000 square feet. The setup will be detailed in Section 4.

Figs. $2 \mathrm{a}$ and $2 \mathrm{~b}$ plot RSS values for all 1-hop neighboring nodes against their distance in two test beds. It shows that monotonic RSS-distance relationship does not hold for the whole network, where RSS varies largely for certain distance. As shown in Fig. 2b, RSS ranges from -60 to $-90 \mathrm{dBm}$ for a 16-foot distance in the 2D network. In addition, a single RSS value may correspond to a wide

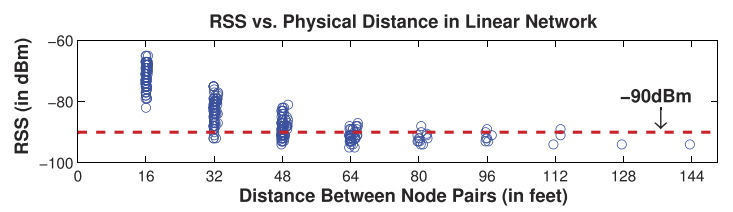

(a) Linear Network: RSS vs. Physical Distance

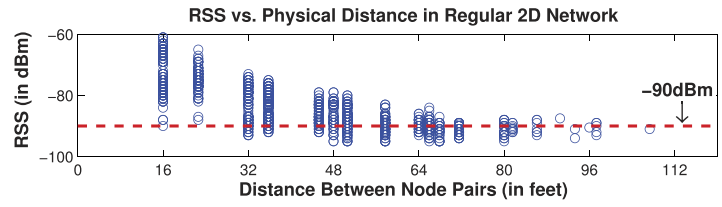

(b) 2D Regular Network: RSS vs. Physical Distance

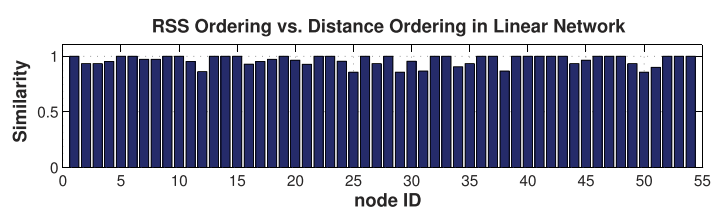

(c) Linear Network: Ordering Similarity

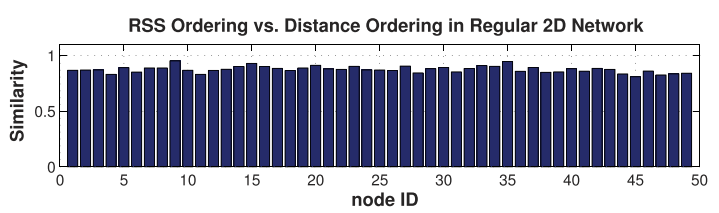

(d) 2D Regular Network: Ordering Similarity

Fig. 2. Empirical data from large-scale experiments.

range of distances. In Fig. 2c, $-90 \mathrm{dBm}$ ranges from 32 to 112 feet in the linear network; even worse, $-90 \mathrm{dBm}$ covers the entire distance spectrum in Fig. $2 b$.

Examining the data from viewpoint of a single node states a different story. For any node $u_{i}$, we can obtain an ordered node list, say $A$, by listing $u_{i}$ 's 1-hop neighbors according to their RSS values sensed at $u_{i}$ by decreasing order; and another node list, say $B$, by ordering its neighbors by increasing distance. Ideally, if RSS decreases monotonically with increasing distance, $A$ and $B$ should be identical. We define similarity between $A$ and $B$ as the percentage of accordant node pairs between them. For example, let $A=$ $\left(u_{1}, u_{2}, u_{3}\right)$ and $B=\left(u_{1}, u_{3}, u_{2}\right)$; then $\left\{u_{1}, u_{2}\right\}$ is an accordant node pair because $u_{1}$ is ordered ahead of $u_{2}$ in both $A$ and $B$, while $\left\{u_{2}, u_{3}\right\}$ is not for its order reverse from $A$ to $B$. We can see that if $A$ and $B$ are roughly consistent with similarity close to 1 , the monotonic feature holds.

Figs. $2 \mathrm{c}$ and $2 \mathrm{~d}$ give similarity results in two test beds. Fig. 2c states that in the linear network, nodes have similarity values close to 1 (the min, mean, and max similarities are $0.86,0.96$, and 1 , respectively), meaning that from single node's point of view, RSS values are approximately monotonic with the distance. This finding also holds for the 2D network as shown in Fig. $2 \mathrm{~d}$.

The above experiments confirm that the monotonic RSSdistance relationship does not hold at the network level, but approximately holds at individual node level. We provide further analysis and discussion concerning this observation in Appendix 2, which can be found on the Computer Society Digital Library at http://doi.ieeecomputersociety. org/10.1109/TPDS.2011.105. 


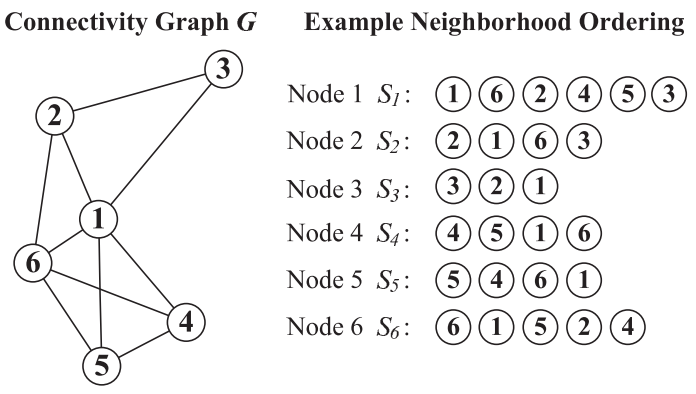

Fig. 3. Neighborhood ordering.

\section{DESIGN}

We consider that nodes use identical RF power configurations during the localization phase. Note that this condition does not prevent any forms of power optimization afterward to prolong the network lifetime.

\subsection{Neighborhood Ordering as a Signature}

Given RSS sensing results for neighboring nodes, a node can obtain a neighborhood ordering with two steps:

- sort its 1-hop neighbors according to their signal strength by decreasing order, and

- add itself as the first element of the sorted node list. Fig. 3 gives an example. Graph $G$ on the left depicts the layout and connectivity of a network. On the right, each node creates a node list starting with itself and containing its 1-hop neighbors ordered by decreasing RSS, i.e., by increasing distance in the ideal case. In general, for any node $u_{i}$, we consider its neighborhood ordering $S_{i}$ as a highdimensional signature in the network. $S_{i}$ has a vector format with three important features:

- $S_{i}$ is unique for each node $u_{i}$.

- $S_{i}$ is position dependent. $S_{i}$ embeds location-related information on both connectivity and proximity.

- $S_{i}$ is obtained without ranging efforts.

For the sake of clarity, in the following, we first use ideal neighborhood orderings for conveying ideas. Namely, $S_{i}$ is consistent with the ordering according to physical distance. Later sections will verify the effectiveness of our design in practical noisy scenarios through both test-bed and simulation experiments.

\subsection{SD: Signature Distance}

This section explains the concept of signature distance, i.e., $\mathrm{SD}$, that quantifies the difference among nodes' signatures to capture proximity relationships.

\subsubsection{Formation, Definition, and Calculation of $S D$}

Say that a pair of nodes $u_{m}$ and $u_{n}$ get flipped between two signatures $S_{i}$ and $S_{j}$, if the ordering of $\left\{u_{m}, u_{n}\right\}$ in $S_{i}$ gets reversed in $S_{j}$. For example, as shown in Fig. 4 , ordered $\{1,6\}$ in $S_{2}=(2,1,6,3)$ gets reversed to $\{6,1\}$ in $S_{5}=(5,4,6,1)$. There are potentially three types of node-pair flips between

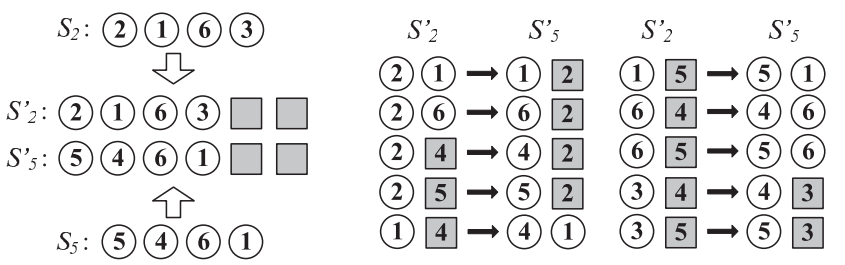

Fig. 5. Ten implicit node-pair flips.

two signatures: 1) explicit flip, 2) implicit flip, and 3) possible flip. If $u_{m}$ and $u_{n}$ appear in both $S_{i}$ and $S_{j}$, we can easily tell whether this node pair gets flipped or not, thus named explicit flip as shown in Fig. 4. Implicit and possible flips are more tricky and explained in the following with examples.

As shown in the left part of Fig. 5, since $S_{2}$ and $S_{5}$ have different sets of node elements, many node pairs in $S_{2}$ do not have their counterparts in $S_{5}$, vice versa. For instance, $\{2,1\},\{2,6\}$ in $S_{2}$ have no related node pairs in $S_{5}$. We solve this problem by attaching "wildcards" to signatures, depicted by gray squares " $\square$ " in the figure. Formally, for $S_{i}$ and $S_{j}$, a number of $\left|S_{i} \cup S_{j}-S_{i}\right|$ wildcards are attached to $S_{i}$ to make $S_{i}^{\prime}$. In $S_{i}^{\prime}$, each wildcard can stand for any node $u \in S_{j}$, but $\notin S_{i}$, i.e., $\forall u \in\left(S_{i} \cup S_{j}-S_{i}\right)$. For example, a gray square in $S_{2}^{\prime}$ can stand for 5 or 4 , and a gray square in $S_{5}^{\prime}$ can be substituted with 2 or 3 . "Wildcard nodes" attached to $S_{i}$ are naturally regarded as further away than neighbors of $u_{i}$ in $S_{i}$, so $S_{i}^{\prime}$ maintains features of a location-dependent signature without violating original proximity relationships in $S_{i}$. Fig. 5 lists all implicit flips from $S_{2}^{\prime}$ to $S_{5}^{\prime}$. We call them implicit because they are not as obvious as explicit flips. For example, node pair $\{2,1\}$ in $S_{2}^{\prime}$ only has a counterpart $\{1, \square\}$ in $S_{5}^{\prime}$, where $\square$ stands for 2 in this case. $\{2,1\} \rightarrow\{1, \square\}$ is a flip since an order reverse occurs no matter which $\square$ in $S_{5}^{\prime}$ stands for 2 .

If a node pair $\left\{u_{m}, u_{n}\right\}$ appears in $S_{i}$, but neither $u_{m}$ nor $u_{n}$ exists in $S_{j}$, we consider it possible that $\left\{u_{m}, u_{n}\right\}$ gets reversed in $S_{j}$. As shown in Fig. 6, $\{2,3\}$ from $S_{2}^{\prime}$ only have a counterpart $\{\square, \square\}$ in $S_{5}^{\prime}$. Without additional information, this node pair gives a possible flip with 50 percent probability. From all the above, the signature distance between $S_{i}$ and $S_{j}$ is defined as follows.

Definition. The signature distance $S D\left(S_{i}, S_{j}\right)$ equals the summation of the number of explicit flips $F_{e}\left(S_{i}, S_{j}\right)$, implicit flips $F_{i}\left(S_{i}, S_{j}\right)$, and possible flips $F_{p}\left(S_{i}, S_{j}\right)$ times 0.5 (i.e., 50 percent probability of flip), namely,

$$
S D\left(S_{i}, S_{j}\right)=F_{e}\left(S_{i}, S_{j}\right)+F_{i}\left(S_{i}, S_{j}\right)+F_{p}\left(S_{i}, S_{j}\right) \times 0.5 .
$$

Take previous $S_{2}$ and $S_{5}$ as an example, $F_{e}\left(S_{2}, S_{5}\right)=1$ in Fig. $4, F_{i}\left(S_{2}, S_{5}\right)=10$ in Fig. 5 , and $F_{p}\left(S_{2}, S_{5}\right)=2$ in Fig. 6 . Thus, we have $S D\left(S_{2}, S_{5}\right)=1+10+2 \times 0.5=12$.

An algorithm for SD calculation is given in Appendix 3, which can be found on the Computer Society Digital Library at http://doi.ieeecomputersociety.org/10.1109/ TPDS.2011.105. Note that $S D\left(S_{i}, S_{j}\right) \equiv S D\left(S_{j}, S_{i}\right)$, because

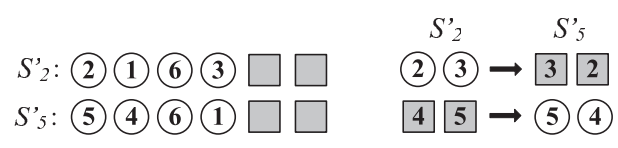

Fig. 4. One explicit node-pair flip.

Fig. 6. Two possible node-pair flips. 


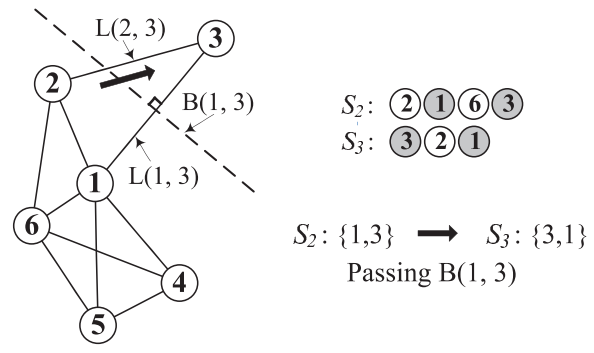

Fig. 7. The insight of a node-pair flip.

each node-pair flip from $S_{i}$ to $S_{j}$ corresponds to one and only one node-pair flip from $S_{j}$ to $S_{i}$.

\subsubsection{Insights into the Signature Distance}

In a signature, an ordered node pair essentially carries a proximity relationship. For example, in Fig. 7 an ordered node pair $\{1,3\}$ in $S_{2}=(2,1,6,3)$ indicates that from node 2 's point of view, node 1 is closer than node 3 . In other words, if we divide the map with a dashed line $B(1,3)$, which is the perpendicular bisector of line segment $L(1,3)$ connecting nodes 1 and $3,\{1,3\}$ in $S_{2}$ implies that node 2 locates on the left side of $B(1,3)$.

Following this example, we can see that a node-pair flip between two signatures corresponds to passing a bisector line in the map, or formally,

Observation 1. A node-pair flip $\left\{u_{m}, u_{n}\right\} \Rightarrow\left\{u_{n}, u_{m}\right\}$ from

$S_{i}$ to $S_{j}$ indicates that the line segment $L\left(u_{i}, u_{j}\right)$ passes

the perpendicular bisector $B\left(u_{m}, u_{n}\right)$.

Additional examples and explanations for this observations are provided in Appendix 4, which can be found on the Computer Society Digital Library at http://doi. ieeecomputersociety.org/10.1109/TPDS.2011.105.

On the other hand, based on the definition of signature distance, $S D\left(S_{i}, S_{j}\right)$ evaluates the difference between $S_{i}$ and $S_{j}$ by counting the number of node-pair flips between them. With Observation 1, we can have

Observation 2. $S D\left(S_{i}, S_{j}\right)$ is equivalent to the number of bisector lines we need to pass if going from neighboring node $u_{i}$ to $u_{j}$ along the line segment $L\left(u_{i}, u_{j}\right)$.

Another key observation concerning the physical distance between two neighboring nodes is that

Observation 3. Under statistically uniform bisector distribution, the number of bisectors passed by line segment $L\left(u_{i}, u_{j}\right)$ is approximately proportional to the physical distance between $u_{i}$ and $u_{j}$, denoted as $P D\left(u_{i}, u_{j}\right)$.

The rationale behind Observation 3 is that a longer physical distance provides a higher probability of passing more bisectors in the map. We provide an example in Appendix 5, which can be found on the Computer Society Digital Library at http://doi.ieeecomputersociety.org/10.1109/ TPDS.2011.105, to further elaborate this observation.

Integrating the above three observations, we can obtain a useful heuristic relationship as follows:

$$
S D\left(S_{i}, S_{j}\right) \propto P D\left(u_{i}, u_{j}\right),
$$

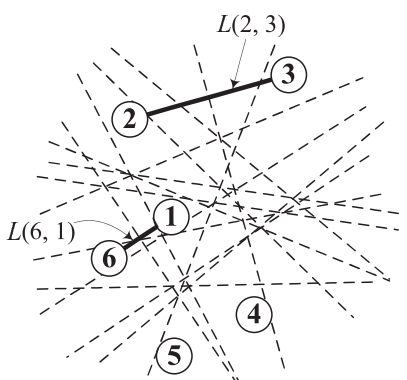

(a) Local Bisector Density

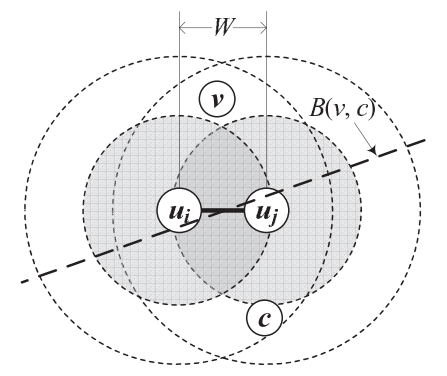

(b) Neighborhood Size
Fig. 8. Caveat 1: local bisector line density.

which says that for neighboring nodes $u_{i}$ and $u_{j}$, their signature distance $S D\left(S_{i}, S_{j}\right)$ is approximately proportional to their physical distance $P D\left(u_{i}, u_{j}\right)$.

\subsection{Two Caveats for the Signature Distance}

There are two remarks for (2): 1) the density and existence of bisectors need to be considered, and 2) the signature distance has only 1-hop effective range.

\subsubsection{Density and Existence of Bisectors}

In Fig. $8 \mathrm{a}, L(2,3)$ passes four bisectors and $L(6,1)$ intersects three. However, $L(2,3)$ is more than twice longer than $L(6,1)$. This inconsistency is caused by spatially unbalanced bisector density in the map. The area around node 1 has a higher bisector density than that of boundary areas near nodes 2 and 3 . This example shows that SD needs to be refined based on local bisector density.

On the other hand, for certain physical distance, calculated signature distances could be different depending on the neighborhood size that determines the existence of bisectors in the map. For example, in Fig. 8b, the physical distance between $u_{i}$ and $u_{j}$ is $W$. If they have a large 1-hop radio range depicted by two big circles, both of them have nodes $v$ and $c$ as neighbors, and $S D\left(S_{i}, S_{j}\right)$ counts the passing of bisector $B(v, c)$. However, if $u_{i}$ and $u_{j}$ have a smaller 1-hop range (due to ambient noise) denoted by gray-filled circles, $v$ and $u$ are absent from $S_{i}$ and $S_{j}$, and $S D\left(S_{i}, S_{j}\right)$ gives a smaller value without counting $B(v, c)$. In fact, the signature distance is a relative value with respect to the dimension of neighborhood area that affects the number of bisectors in the map.

\subsubsection{1-Hop Effective Range}

Another caveat on (2) is that it may not hold for nonneighboring node pairs. Fig. 9 shows an example, where nodes $u_{i}, u_{j}$, and $u_{k}$ are located far from each other, and their neighboring areas (denoted with dashed circles) do not overlap. In this case, $S D\left(S_{i}, S_{j}\right)$ and $S D\left(S_{i}, S_{k}\right)$ only count possible flips determined by the number of nodes in

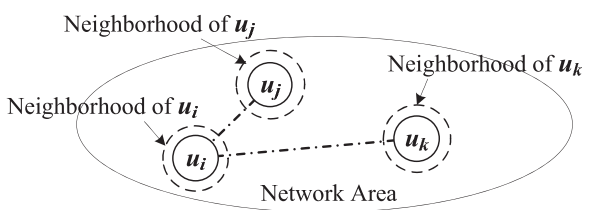

Fig. 9. The case of far-away node pairs. 
$S_{i}, S_{j}$, and $S_{k}$. Under similar 1-hop radio range, $S D\left(S_{i}, S_{j}\right)$ and $S D\left(S_{i}, S_{k}\right)$ could have similar values, although the physical distances of two node pairs are dramatically different. In fact, the heuristic relationship in (2) is not effective beyond 1-hop range.

\subsection{RSD: Regulated Signature Distance}

To address aforementioned caveats, this section proposes the regulated signature distance, i.e., RSD, as a refined version of the SD design. For 1-hop neighboring nodes $u_{i}$ and $u_{j}$, their RSD is defined as follows:

$$
R S D\left(u_{i}, u_{j}\right)=S D\left(S_{i}, S_{j}\right) \cdot \frac{\sqrt{K}}{K(K-1) / 2} .
$$

Equation (3) refines $S D\left(S_{i}, S_{j}\right)$ with a factor $\sqrt{K} /$ $(K(K-1) / 2)$, where $K=\left|S_{i} \cup S_{j}\right|$ is the number of nodes in the neighborhood of $u_{i}$ and $u_{j}$ combined. $K(K-1) / 2$ is used to normalize $S D\left(S_{i}, S_{j}\right)$ with the local bisector density, and $\sqrt{K}$ estimates the diameter of this neighborhood, putting the factor of neighborhood size into consideration. We provide detailed derivation for (3) in Appendix 6, which can be found on the Computer Society Digital Library at http://doi.ieee computersociety.org/10.1109/TPDS. 2011.105.

To address multihop nodes in the network, we propose a more generic definition for RSD as follows:

- for 1-hop neighboring nodes $u_{i}$ and $u_{j}, R S D\left(u_{i}, u_{j}\right)$ is directly computed with (3); and

- $\quad$ for nonneighboring nodes $u_{i}$ and $u_{j}, R S D\left(u_{i}, u_{j}\right)$ is calculated as the smallest accumulated RSD from all paths between $u_{i}$ and $u_{j}$.

Here, the accumulated RSD of a path from node $u_{i}$ to $u_{j}$ is computed as the summation of RSD values of all 1-hop node pairs along this path.

\subsection{Design Embedding}

With local RSS sensing results, RSD can be calculated at each node or in a localization server. For connectivity-based localization algorithms such as MDS-MAP [24], DV-Hop [25], and RPA [31], applying RSD is convenient and incurs little overhead. Without modifying the major design of original algorithm, the RSD value can be used instead of "1" (indicating connection) for neighboring nodes. Specifically, in MDS-MAP [24], everything remains the same except that the distance matrix now holds the smallest accumulated RSD values instead of the least number of hops among nodes in the network. For DV-Hop [25], similarly, the relative distance can be evaluated with the smallest accumulated RSD instead of shortest path hops. And the expected physical distance for 1 unit of RSD can be calculated with

$$
R S D_{\text {unit }}=\frac{\sum_{i \neq j} \operatorname{Distance}\left(v_{i}, v_{j}\right)}{\sum_{i \neq j} R S D\left(v_{i}, v_{j}\right)} .
$$

For RPA [31], in addition to embedding RSD in the HopTERRAIN step, the refinement step also benefits from applying RSD for local iterative position adjustment. We provide a detailed explanation about these schemes as well as complexity analysis in Appendix 7, which can be found

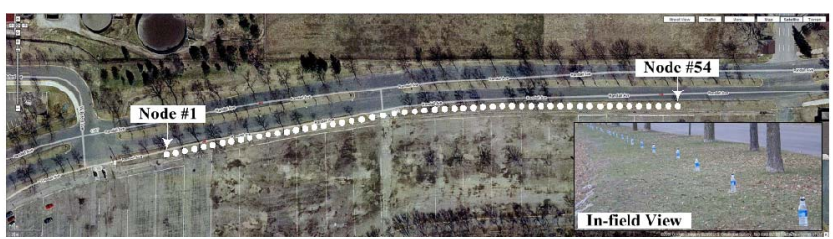

Fig. 10. Test-Bed Experiments I: linear network.

on the Computer Society Digital Library at http:// doi.ieeecomputersociety.org/10.1109/TPDS.2011.105.

\section{Test-Bed Evaluation}

In this section, we report two types of outdoor experiments with 54 and 49 MICAz motes, respectively. We also provide a comprehensive simulation study as Appendix 9, in the supplement part of the paper, which can be found on the Computer Society Digital Library at http:// doi.ieeecomputersociety.org/10.1109/TPDS. 2011.105.

\subsection{Experiment I: Linear Network}

\subsubsection{Test-Bed Setup}

Evaluation starts from a linear topology widely applied in road networks. In Fig. 10, 54 motes were deployed on the grass curb along a road (with surrounding obstacles such as trees, fence, and vehicles). Nodes were left 8 inches above the ground with a 16-foot distance between adjacent nodes. Every node broadcast 100 packets with mechanisms for collision avoidance. The radio TX power was $0 \mathrm{dBm}$ at channel $26\left(F_{c}=2,480 \mathrm{MHz}\right)$ to avoid WiFi interference. Each packet contained the sender ID and a sequence number. When a node received a packet, it logged the RSS, sender ID, and packet sequence number into flash. The linear network is about 848 feet long with 15 hops between node \#1 and node \#54. The maximum 1-hop radio range was about 144 feet and the average 1-hop neighborhood size was 6.7 (nodes).

\subsubsection{Distance Correlations}

Fig. 11 shows correlations between hop-based distance, RSD, and physical distance. Figs. 11a and 11b plot all 1-hop node pairs in the network. In both figures, the $X$-axis denotes the physical distance, and the $Y$-axis denotes the hop-based distance and RSD, respectively. Fig. 11a reveals that 1-hop node pairs have an identical hop distance of 1 , and thus, the correlation coefficient is $\rho=0$ within 1-hop range. On the contrary, in Fig. $11 \mathrm{~b}$ node pairs with different distances mostly can be differentiated from each other based on their RSD values $(\rho=0.89)$. Therefore, we conclude that RSD owns a subhop resolution that is not available from traditional hop-based distances.

Figs. 11c and 11d plot node pairs (1-hop and multihop) according to their hop distance, RSD, and physical distance. Although correlation coefficients are close in this case ( $\rho=0.98$ for hop versus $\rho=0.99$ for RSD), RSD provides a better resolution. In Fig. 11c, a physical distance can only be mapped to an integer hop count, while in Fig. 11d, the mapping is continuous. Localization results will show that RSD's subhop resolution can solve an ambiguity problem explained in the following. 


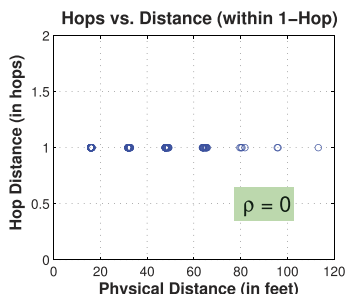

(a)

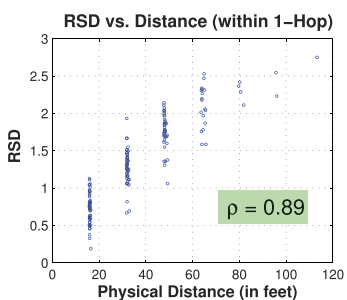

(b)

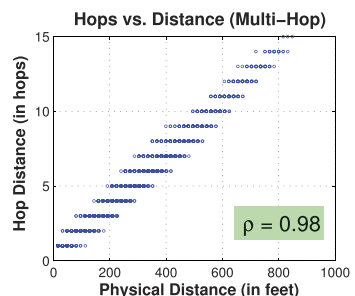

(c)

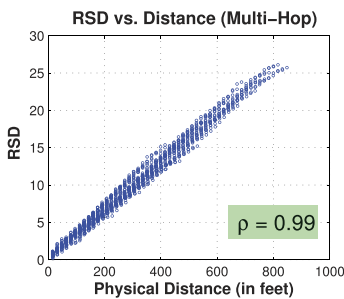

(d)

Fig. 11. Distance correlation comparison: RSD versus Hop (linear network).
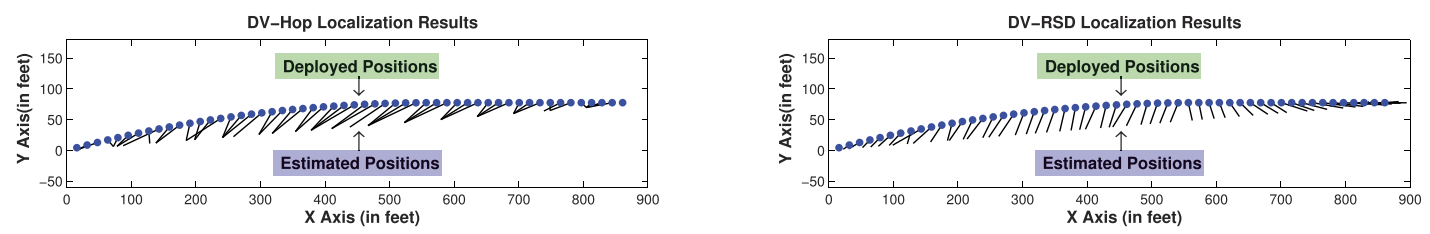

(a) DV-Hop vs. DV-RSD
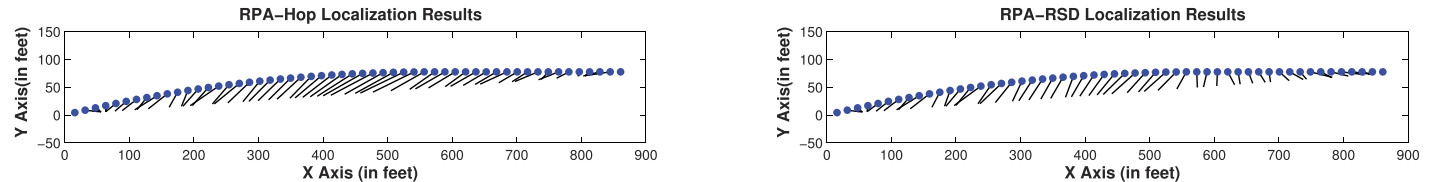

(b) RPA-Hop vs. RPA-RSD
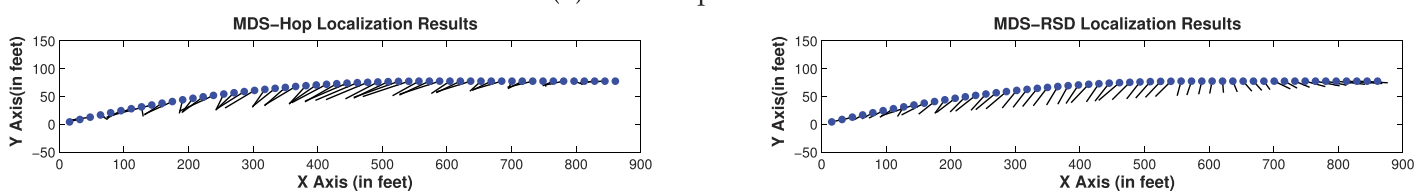

(c) MDS-Hop vs. MDS-RSD

Fig. 12. Localization in linear networks: hop-based distance versus RSD.

\subsubsection{Localization Performance}

We use terms "MDS-Hop," "DV-Hop," and "RPA-Hop" for original hop-based methods and "MDS-RSD," "DV-RSD," and "RPA-RSD" for corresponding methods embedded with RSD. Let's first look at DV-Hop and DV-RSD. Fig. 12a shows localization results from both algorithms with two terminal nodes of the linear network as anchors. In the figure, black line segments are plotted starting from nodes' deployed positions (blue dots) and pointing to estimated locations. In the left subfigure for DV-Hop, many nodes are mapped to identical estimated locations, i.e., an ambiguity problem. DV-RSD does not encounter this issue as shown in the right subfigure.

Fig. 12b shows results from RPA-Hop and RPA-RSD with two refinement steps. RPA-Hop and RPA-RSD both achieved unique position estimation for each node. For RPA-Hop, this is credited to the refinement step that smooths the estimated position of a node over that of its neighbors, naturally solving the problem of clustered mapping. From Fig. 12b, we can observe that RPA-RSD gives better localization accuracy than RPA-Hop.

Fig. 12c gives results from MDS-Hop and MDS-RSD. We found that 2D MDS is not stable for close-to-linear topology due to the singularity issue in matrix decomposition. Here, 1D MDS is applied and two terminal nodes are used as anchors for map scaling and rotation. Fig. 12c states that
MDS algorithm itself cannot solve the ambiguity problem, while MDS-RSD shows enhanced accuracy without clustered mapping.

We define localization error as the offset from true position to estimated location. Fig. 13 gives error boxplots of six methods. We can see that RSD-embedded methods (“*-RSD") all have reduced errors than their "*-Hop" based versions statistically, where the maximum errors of "MDS-Hop," "DV-Hop," and "RPA-Hop" get reduced by 27, 35, and 29 percent, respectively.

\subsection{Experiment II: Regular 2D Network}

\subsubsection{Test-Bed Setup}

The second test-bed targets a 2D network with grid-shaped topology. Fig. 14 shows the experiment scenario of a parking lot at night. We placed $49 \mathrm{MICAz}$ nodes in a $7 \times 7$ grid,

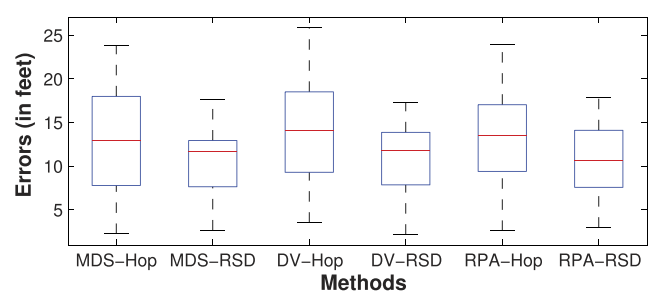

Fig. 13. Comparison: RSD versus Hop distance. 


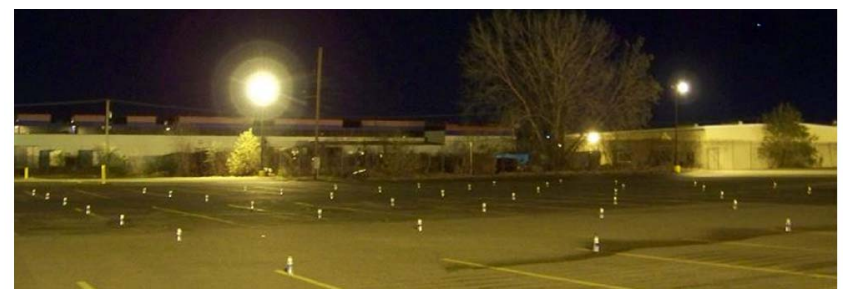

Fig. 14. Test-Bed Experiments II: regular 2D network.

covering an area of about $100 \times 100$ square feet. Rows and columns were about 16 feet apart. Other setups are similar to that in Experiment I. Table 1 lists that it is a 3-hop network. 1-hop radio range varies from 20 to 100 feet among different node pairs.

\subsubsection{Distance Correlations}

Fig. 15 shows correlations between hop-based distance, RSD, and physical distance in the 2D network. Figs. 15a and $15 \mathrm{~b}$ confirm that RSD provides a subhop resolution while hop distance does not. Fig. 15b verifies that for MICAz motes using monopole whip antenna with radiation pattern close to isotropic [54], neighborhood ordering based on RSS can be a heuristic indicator for the "near-far" relationship. For multihop nodes, Figs. 15c and 15d show that RSD owns a higher correlation coefficient than hop-based distance, indicating that RSD is superior as a metric for proximity.

\subsubsection{Localization Performance}

The network has only three hops that is not suitable for DVHop and RPA. To be fair, we only applied MDS-based algorithms in this evaluation. Performance comparisons for DV-Hop and RPA in nonlinear-shape networks were investigated with extensive simulation in Appendix 9, which can be found on the Computer Society Digital Library at http://doi.ieeecomputersociety.org/10.1109/ TPDS.2011.105.

Figs. 16a and 16b show localization results from MDSHop and MDS-RSD with identical four anchor nodes. In both figures, blue dots depict nodes' true positions and line segments point to their estimated positions. Clearly, MDSRSD gives better localization accuracy than MDS-Hop in this case.

To eliminate possible bias caused by anchor selection, we randomly picked different numbers of anchors, from 4 to 10 in step of 1, and tried each for 1,000 runs. Fig. 16c plots averaged
TABLE 1

Statistics of the 2D Network

\begin{tabular}{|c|c|c|c|}
\hline Statistics & 1-Hop & 2-Hop & 3-Hop \\
\hline Number of Node Pairs & 575 & 519 & 82 \\
\hline Minimum Distance (in feet) & 16 & 22.63 & 80 \\
\hline Median Distance (in feet) & 35.78 & 71.55 & 97.32 \\
\hline Maximum Distance (in feet) & 97.32 & 124.96 & 135.76 \\
\hline
\end{tabular}

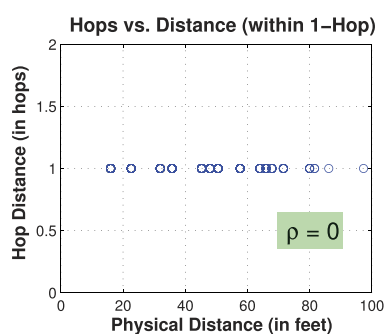

(a)

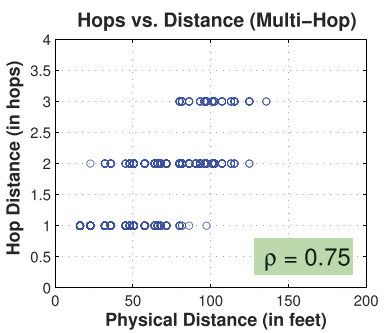

(c)

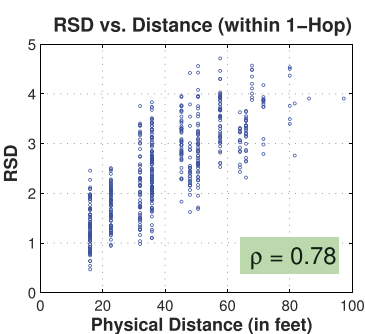

(b)

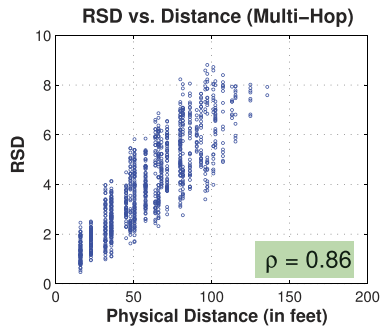

(d)
Fig. 15. Distance correlation comparison: RSD versus Hop (regular 2D network).

max and median errors for both methods, from which we can conclude that 1) MDS-RSD offers a better performance than MDS-Hop across all numbers of anchors, and 2) embedding RSD is more effective than adding more anchors. For example, for four anchors, RSD reduces the maximum and median errors by about 27 and 30 percent, respectively. In addition to the above analysis, we also provide detailed confidence interval analysis for results in Fig. 16c as Appendix 8, which can be found on the Computer Society Digital Library at http://doi.ieeecomputersociety.org/ 10.1109/TPDS.2011.105.

Radio is notorious for irregularity. Nevertheless, our testbed evaluation shows encouraging results that applying RSD allows us to achieve better localization accuracy than considering connectivity alone.

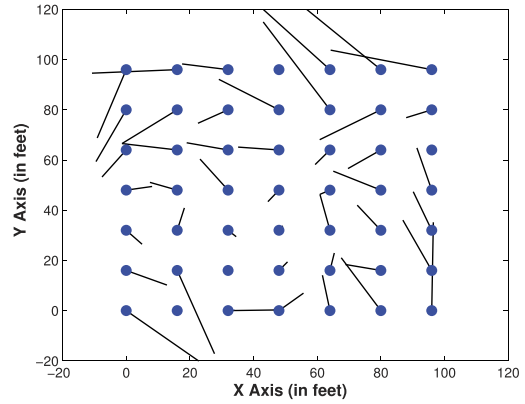

(a) Localization by MDS-Hop

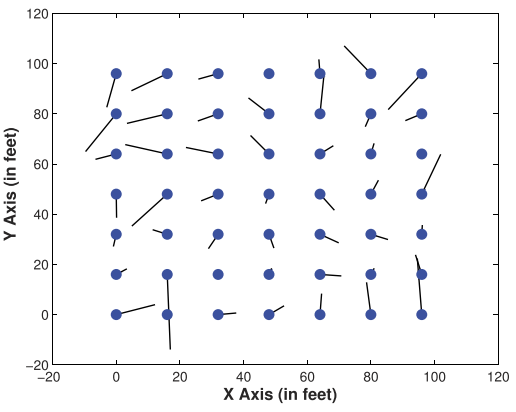

(b) Localization by MDS-RSD

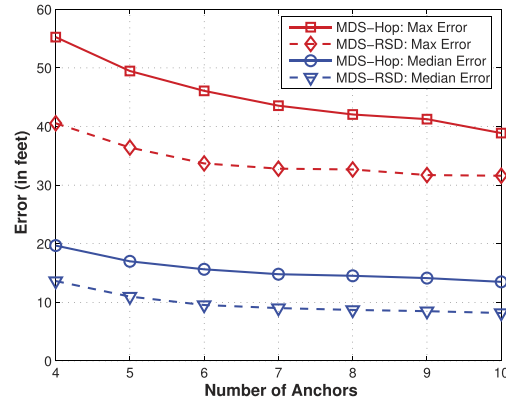

(c) Statistical Comparison

Fig. 16. Localization results of MDS-RSD and MDS-Hop. 


\section{Conclusions}

This paper proposes $R S D$, a proximity metric for achieving range-free localization beyond connectivity. Starting from the concept of neighborhood ordering as a location-dependent signature for each node in the network, we introduce the design of RSD that quantifies the difference between node signatures to capture the distance relationships among neighboring nodes with subhop resolution. With little overhead, RSD can be conveniently embedded in connectivitybased localization algorithms, and our test-bed evaluation demonstrates that applying RSD helps solve the ambiguity problem and considerably improves localization accuracy. In addition, simulation reveals that RSD is effective under different network configurations and offers a nice feature of robustness for unevenly distributed radio path loss.

\section{ACKNOWLEDGMENTS}

This research was partially supported by the US National Science Foundation (NSF) grants CNS-0626609, CNS0626614, and CRI-0708344.

\section{REFERENCES}

[1] D. Culler, D. Estrin, and M. Srivastava, "Guest Editors' Introduction: Overview of Sensor Networks," Computer, vol. 37, no. 8, pp. 41-49, 2004.

[2] G. Simon et al., "Sensor Network-Based Countersniper System," Proc. Second Int'l Conf. Embedded Networked Sensor Systems (SenSys '04), 2004.

[3] G. Werner-Allen, J. Johnson, M. Ruiz, and J. Lees, "Monitoring Volcanic Eruptions with a Wireless Sensor Network," Proc. European Wireless Sensor Networks (EWSN '05), 2005.

[4] A. Terzis et al., "Slip Surface Localization in Wireless Sensor Networks for Landslide Prediction," Proc. Fifth Int'l Conf. Information Processing in Sensor Networks (IPSN '06), 2006.

[5] B. Karp and H.T. Kung, "GPSR: Greedy Perimeter Stateless Routing for Wireless Networks," Proc. MobiCom, 2000.

[6] Y. Kim, R. Govindan, B. Karp, and S. Shenker, "Geographic Routing Made Practical," Proc. Second Conf. Networked Systems Design Implementation (NSDI '05), 2005.

[7] P. Bahl and V.N. Padmanabhan, "Radar: An In-Building RF-Based User Location and Tracking System," Proc. IEEE INFOCOM, 2000.

[8] N.B. Priyantha, A. Chakraborty, and H. Balakrishnan, "The Cricket Location-Support System," Proc. ACM MobiCom, 2000.

[9] X. Cheng, A. Thaeler, G. Xue, and D. Chen, "TPS: A Time-Based Positioning Scheme for Outdoor Wireless Sensor Networks," Proc. IEEE INFOCOM, 2004.

[10] A. Savvides, C. Han, and M.B. Strivastava, "Dynamic FineGrained Localization in Ad-Hoc Networks of Sensors," Proc. ACM MobiCom, 2001.

[11] S. Lanzisera, D.T. Lin, and K.S.J. Pister, "RF Time of Flight Ranging for Wireless Sensor Network Localization," Proc. Workshop on Intelligent Solutions in Embedded Systems (WISES '06), 2006.

[12] J. Liu, Y. Zhang, and F. Zhao, "Robust Distributed Node Localization with Error Management," Proc. Seventh ACM Int'l Symp. Mobile Ad Hoc Networking and Computing (MobiHoc), 2006.

[13] D. Moore, J. Leonard, and K.S.J. Pister, "Robust Distributed Network Localization with Noisy Range Measurements," Proc. Second Int'l Conf. Embedded Networked Sensor Systems (SenSys '04), 2004.

[14] X. Cheng, H. Shu, Q. Liang, and D.H.-C. Du, "Silent Positioning in Underwater Acoustic Sensor Networks," IEEE Trans. Vehicular Technology, vol. 57, no. 3, pp. 1756-1766, May 2008.

[15] M. Maróti, B. Kusý, G. Balogh, P. Völgyesi, A. Nádas, K. Molnár, S. Dóra, and Á. Ledeczi, "Radio Interferometric Geolocation," Proc. Third Int'l Conf. Embedded Networked Sensor Systems (SenSys '05), 2005.

[16] H. Chang, J. Tian, T. Lai, H. Chu, and P. Huang, "Spinning Beacons for Precise Indoor Localization," Proc. Sixth ACM Conf. Embedded Network Sensor Systems (SenSys '08), 2008.
[17] A. Basu et al., "Distributed Localization by Noisy Distance and Angle Information," Proc. Seventh ACM Int'l Symp. Mobile Ad Hoc Networking and Computing (MobiHoc), 2006.

[18] Z. Yang and Y. Liu, "Quality of Trilateration: Confidence-Based Iterative Localization," Proc. Int'l Conf. Distributed Computing Systems (ICDCS '08), 2008.

[19] K. Chintalapudi, R. Govindan, R. Govindan, and G. Sukhatme, "Ad-Hoc Localization Using Ranging and Sectoring," Proc. IEEE INFOCOM, 2004.

[20] D. Niculescu and B. Nath, "Ad Hoc Positioning System (APS) Using AOA," Proc. IEEE INFOCOM, 2003.

[21] J. Bruck, J. Gao, and A. Jiang, "Localization and Routing in Sensor Networks by Local Angle Information," Proc. Sixth ACM Int'l Symp. Mobile Ad Hoc Networking and Computing (MobiHoc), 2005.

[22] D.K. Goldenberg et al., "Localization in Sparse Networks Using Sweeps," Proc. ACM MobiCom, 2006.

[23] N. Bulusu, J. Heidemann, and D. Estrin, "GPS-Less Low Cost Outdoor Localization for Very Small Devices," IEEE Personal Comm. Magazine, vol. 7, no. 5, pp. 28-34, Oct. 2000.

[24] Y. Shang, W. Ruml, Y. Zhang, and M.P.J. Fromherz, "Localization from Mere Connectivity," Proc. Fourth ACM Int'l Symp. Mobile Ad Hoc Networking and Computing (MobiHoc), 2003.

[25] D. Niculescu and B. Nath, "DV Based Positioning in Ad Hoc Networks," J. Telecomm. Systems, vol. 22, no. 4, pp. 267-280, 2003.

[26] T. He, C. Huang, B.M. Blum, and J.A. Stankovic, "Range-Free Localization Schemes in Large-Scale Sensor Networks," Proc. ACM MobiCom, 2003.

[27] S. Lederer, Y. Wang, and J. Gao, "Connectivity-Based Localization of Large Scale Sensor Networks with Complex Shape," Proc. INFOCOM, 2003.

[28] N. Bulusu, J. Heidemann, D. Estrin, and T. Tran, "Self-Configuring Localization Systems: Design and Experimental Evaluation," Trans. ACM Embedded Computing Systems, vol. 3, no. 1, 2004.

[29] M. Li and Y. Liu, "Rendered Path: Range-Free Localization in Anisotropic Sensor Networks with Holes," Proc. ACM MobiCom, 2007.

[30] R. Nagpal, H. Shrobe, and J. Bachrach, "Organizing a Global Coordinate System from Local Information on an Ad Hoc Sensor Network," Proc. Second Int'l Conf. Information Processing in Sensor Networks (IPSN '03), 2003.

[31] C. Savarese, J.M. Rabaey, and K. Langendoen, "Robust Positioning Algorithms for Distributed Ad-Hoc Wireless Sensor Networks," Proc. General Track of the Ann. Conf. USENIX, 2002.

[32] C. Wang and L. Xiao, "Locating Sensors in Concave Environments," Proc. IEEE INFOCOM, 2006.

[33] L. Doherty, K.S.J. Pister, and L. El Ghaoui, "Convex Position Estimation in Wireless Sensor Networks," Proc. IEEE INFOCOM, 2001.

[34] L. Römer, "The Lighthouse Location System for Smart Dust," Proc. First Int'l Conf. Mobile Systems (MobiSys '03), 2003.

[35] R. Stoleru et al., "A High-Accuracy, Low-Cost Localization System for Wireless Sensor Networks," Proc. Third Int'l Conf. Embedded Networked Sensor Systems (SenSys '05), 2005

[36] Z. Zhong and T. He, "MSP: Multi-Sequence Positioning of Wireless Sensor Nodes," Proc. Fifth Int'l Conf. Embedded Networked Sensor Systems (SenSys '07), 2007.

[37] K. Whitehouse et al., "The Effects of Ranging Noise on Multihop Localization: An Empirical Study," Proc. Fourth Int'l Symp. Information Processing in Sensor Networks (IPSN '05), 2005.

[38] K. Whitehouse, C. Karlof, and D. Culler, "A Practical Evaluation of Radio Signal Strength for Ranging-Based Localization," ACM Mobile Computing and Comm. Rev. (SIGMOBILE), vol. 11, no. 1, p. $41,2007$.

[39] X. Li, H. Shi, and Y. Shang, "A Partial-Range-Aware Localization Algorithm for Ad-Hoc Wireless Sensor Networks," Proc. IEEE 29th Ann. Int'l Conf. Local Computer Networks (LCN '04), 2004.

[40] X. Li, H. Shi, and Y. Shang, "A Sorted RSSI Quantization Based Algorithm for Sensor Network Localization," Inter. J. Ad Hoc and Ubiquitous Computing, vol. 1, no. 4, pp. 222-229, 2006.

[41] K. Srinivasan, "Understanding the Causes of Packet Delivery Success and Failure in Dense Wireless Sensor Networks," Proc. Fourth Int'l Conf. Embedded Networked Sensor Systems, 2006.

[42] E. Miluzzo, X. Zheng, K. Fodor, and A.T. Campbell, "Radio Characterization of 802.15.4 and Its Impact on the Design of Mobile Sensor Networks," Proc. Fifth European Conf. Wireless Sensor Networks (EWSN '08), 2008. 
[43] J.A. Costa, N. Patwari, and A.O. Hero III, "Distributed WeightedMultidimensional Scaling for Node Localization in Sensor Networks," Trans. ACM Sensor Networks, vol. 2, no. 1, pp. 39-64, 2006.

[44] N. Patwari, A.O. Hero Iii, and J.A. Costa, "Learning Sensor Location from Signal Strength and Connectivity," Advances in Information Security Series, Springer, 2006.

[45] J. Hightower, G. Borriello, and R. Want, "SpotON: An Indoor 3D Location Sensing Technology Based on RF Signal Strength," Proc. IEEE Int'l Conf. Computational Science and Eng. (CSE), 2000.

[46] Z. Zhong, D. Wang, and T. He, "Sensor Node Localization Using Uncontrolled Events," Proc. Int'l Conf. Distributed Computing Systems (ICDCS '08), 2008.

[47] A. Varshavsky, E. Delara, J. Hightower, A. Lamarca, and V. Otsason, "GSM Indoor Localization," Pervasive and Mobile Computing, vol. 3, no. 6, pp. 698-720, 2007.

[48] T.S. Rappaport, Wireless Communications, Principles and Practice. Prentice Hall, 1996.

[49] K. Yedavalli and B. Krishnamachari, "Sequence-Based Localization in Wireless Sensor Networks," Trans. IEEE Mobile Computing, vol. 7, no. 1, pp. 81-94, Jan. 2008.

[50] Y. Zhang, L. Zhang, and X. Shan, "Ranking-Based Statistical Localization for Wireless Sensor Networks," Proc. IEEE Wireless Comm. and Networking Conf. (WCNC'08), 2008.

[51] E.W. Weisstein, "Circle Division by Lines," MathWorld http:// mathworld.wolfram.com/CircleDivisionbyLines.html, 2011.

[52] S. Carlsson, "A Variant of Heapsort with Almost Optimal Number of Comparisons," Information Processing Letters, vol. 24, no. 4, pp. 247-250, 1987.

[53] M.J. Greenacre, Theory and Applications of Correspondence Analysis. Academic Press, Inc., 1984.

[54] K. Smith, "Antennas for Low Power Applications," RFM Corp., Dallas. http://www.rfm.com/corp/appdata/antenna.pdf, 2011.

[55] The ns Manual, Chapter 18: Radio Propagation Models. ed.: Kevin Fall et al., http://www.isi.edu/nsnam/ns/doc/index.html, 2011.

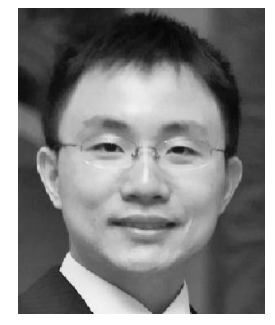

Ziguo Zhong received the BSEE and MSEE degrees from the Department of Radio Engineering at Southeast University, Nanjing, China, in 2003 and 2005, respectively. He received the $\mathrm{PhD}$ degree in computer science from the University of Minnesota-Twin Cities in 2010. His research interests include embedded and distributed systems, computer networking, energy aware and mobile comdigital signal processing, and robotics.

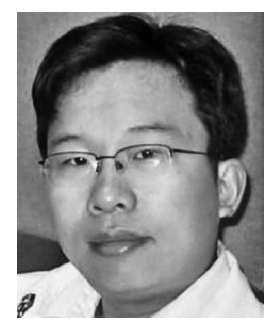

Tian $\mathrm{He}$ is an associate professor with the Department of Computer Science and Engineering, University of Minnesota-Twin Cities. He is the author and coauthor of more than 100 papers in premier journals and conferences with more than 5,000 citations. As a recipient of the US National Science Foundation (NSF) CAREER Award '09 and McKnight Land-Grand Professorship '09-'11, he served a few program chair position in international conferences, and also serves as an editorial member for several international journals including ACM TOSN. His research includes wireless sensor networks, intelligent transportation systems, real-time embedded systems, and distributed systems. He is a member of the IEEE.

$\triangleright$ For more information on this or any other computing topic, please visit our Digital Library at www.computer.org/publications/dlib. 